\title{
Phenomenology of CR-scattering on pre-existing MHD modes
}

\section{Ottavio Fornieri, ${ }^{a * *}$ Daniele Gaggero, ${ }^{b}$ Silvio Sergio Cerri, ${ }^{c}$ Pedro De La Torre Luque $^{d}$ and Stefano Gabici ${ }^{e}$}

${ }^{a}$ Deutsches Elektronen-Synchrotron (DESY), Platanenallee 6, D-15738 Zeuthen, Germany

${ }^{b}$ Instituto de Física Teórica UAM-CSIC, Campus de Cantoblanco, E-28049 Madrid, Spain

${ }^{c}$ Department of Astrophysical Sciences, Princeton University, Princeton, NJ, United States

${ }^{d}$ The Oskar Klein Centre, Department of Physics, Stockholm University, AlbaNova SE-10691 Stockholm, Sweden

e Université de Paris, CNRS, Astroparticule et Cosmologie, F-75006 Paris, France

E-mail: ottavio.fornieri@desy.de

We present the phenomenological implications of the micro-physics of CR diffusion as resulting from particle scattering onto the three modes in which Magneto-Hydro-Dynamics (MHD) cascades are decomposed. We calculate the diffusion coefficients from first principles based on reasonable choices of the physical quantities characterizing the different environments of our Galaxy, namely the Halo and the Warm Ionized Medium, and implement for the first time these coefficients in the DRAGON2 numerical code. Remarkably, we obtain the correct propagated slope and normalization for all the charged species taken into account, without any ad-hoc tuning of the transport coefficients. We show that fast magnetosonic modes dominate CR confinement up to $\sim 100 \mathrm{TeV}$; Alfvénic modes are strongly subdominant due to the anistropy of the cascade (in agreement with previous findings) up to rigidities in the sub-PeV domain, where their contribution may show up as a spectral feature, potentially observable in the upcoming years. We also find that such framework cannot be responsible for CR confinement below $\sim 200 \mathrm{GeV}$, possibly leaving room for an additional confinement mechanism, and that the Kolmogorov-like scaling of the $B / C$ ratio cannot be reproduced. Therefore this scaling might not be the imprint of the pre-exisiting turbulence spectrum.

$37^{\text {th }}$ International Cosmic Ray Conference (ICRC 2021)

July 12th - 23rd, 2021

Online - Berlin, Germany

\footnotetext{
${ }^{*}$ Presenter
} 


\section{Introduction}

A diverse set of data has suggested for more than 50 years the existence of a non-thermal population of cosmic particles confined in our Galaxy for a characteristic timescale of $\sim 10^{7}$ years (see [1] and references therein), much larger than the crossing time of the Galactic halo associated to a free-streaming particle, thus suggesting a diffusive transport rather than a ballistic motion.

The canonical framework to interpret particle diffusion in the Interstellar Medium is the socalled quasi-linear theory (QLT), able to predict particle confinement as a function of rigidity due to the interaction with isotropic, transverse magnetic fluctuations $[2,3]$. This picture has inspired most of the phenomenological interpretations of the cosmic-ray (CR) diffuse emission, computed by solving the so-called transport equation with numerical or semi-analytical methods, such as GALPROP $^{1}$ or DRAGON ${ }^{2}$. In particular, the DRAGON package has provided significant steps forward in the context of inhomogeneous CR transport, by moving away from the zero-order modeling of isotropic, homogeneous diffusion and implementing in some contexts independent parallel and perpendicular scalings $\left(D_{\|} \neq D_{\perp}\right)$, as well as position-dependent diffusion coefficients.

Supporting the importance of such inhomogeneous paradigm, in [4] different scaling relations of the diffusion coefficient in the Galactic disk and in the halo were invoked to explain the hardening in the proton spectrum (first discovered by PAMELA and then characterized by AMS-02 with large accuracy) for a peculiar combination of parameters in the disk and halo. These studies have provided a precious insight on the impact of the transport properties on the CR observables, although they do not contain a theoretical explanation of the microphysics of the particle-wave interactions.

From a modern understanding the MHD turbulence, this can be decomposed into a mixed cascade of (incompressible) Alfvénic fluctuations, and (compressible) slow and fast magnetosonic fluctuations, as theoretically demonstrated and numerically confirmed by simulations $[5,6]$. Regarding the Alfvénic component, a reference scenario is the model put forward by Goldreich and Sridhar [7] (hereafter GS95), that stems from the observation that mixing field lines, in directions perpendicular to the regular magnetic field on a hydrodynamical-like timescale, is easier than bending them, because of the magnetic tension. This perpendicular mixing is able to couple waves that travel along the regular field, obeying a critical balance condition: $k_{\|} v_{A} \sim k_{\perp} u_{k}$. As a result, Alfvén modes are progressively more anisotropic as the cascade goes on, a behaviour that is captured by the scaling relations $E_{A}\left(k_{\perp}\right) \propto k_{\perp}^{-5 / 3}$ and $k_{\|} \propto k_{\perp}^{2 / 3}$. Same behaviour holds for slow modes, while a isotropic cascade is exhibited by the fast modes, with an energy spectrum $E_{M}(k) \propto k^{-3 / 2}$.

From the point of view of the environment, all the relevant phases of the interstellar medium (ISM) can be approximated as a low-beta plasma $\left(\beta=P_{\mathrm{g}} / P_{\mathrm{B}}\right.$, with $P_{\mathrm{g}}$ the plasma thermal pressure and $P_{\mathrm{B}}$ the magnetic pressure) in almost all cases [8]. In this regime, fast-magnetosonic modes are less damped than Alfvénic fluctuations [see 9, and references therein].

As a consequence of this paradigm, the picture of the microphysics of cosmic-ray pitch-angle scattering may be deeply revised. As shown in [10], the cosmic-ray scattering rates, evaluated for the GS95 highly anisotropic Alfvénic spectrum, significantly decrease with respect to the simple assumption of isotropic cascade. Conversely, the isotropy of the fast-magnetosonic cascade may allow these modes to dominate CR scattering for most of the pitch angle range [17]. A (weakly) non-

${ }^{1}$ https://galprop.stanford.edu/

${ }^{2}$ https://github.com/cosmicrays 
linear modification of the QLT theory of CR scattering in MHD turbulence has been developed e.g. in $[11,12]$, and then further reconsidered in [13] to explicitly address the role of fast-magnetosonic modes. Based on the above-mentioned modified-QLT framework, we consistently compute the diffusion coefficients for a wide rigidity range in both environments, and depending on several parameters, including the plasma $\beta$ and the amplitude of the injected turbulent fluctuations. We then implement these coefficients in the DRAGON2 numerical package and test the predictions of the theory against the most recent data provided by the AMS-02 Collaboration.

\section{Scattering rate and diffusion coefficient in MHD turbulence}

To address the contributions to the scattering efficiency arising from the different MHD cascades (namely, Alfvénic and fast/slow magnetosonic), we follow the approach based on the mentioned non-linear extension of the original QLT. We remind to [14] for the detailed calculations.

In this formalism, a particle with velocity $v$ forming an angle $\theta$ with the background magnetic field $\boldsymbol{B}_{0}$ (i.e. pitch angle $\mu \equiv v_{\|} /|\boldsymbol{v}|=\cos \theta$ ) propagating in a turbulent environment with power spectrum described by $I$, exhibits a scattering rate in pitch-angle space that is expressed as $[12,15]$ :

$$
D_{\mu \mu}=\Omega^{2}\left(1-\mu^{2}\right) \int d^{3} \boldsymbol{k} \sum_{n=-\infty}^{+\infty} R_{n}\left(k_{\|} v_{\|}-\omega+n \Omega\right)\left[\frac{n^{2} J_{n}^{2}(z)}{z^{2}} I^{\mathrm{A}}(\boldsymbol{k})+\frac{k_{\|}^{2}}{k^{2}} J_{n}^{\prime 2}(z) I^{\mathrm{M}}(\boldsymbol{k})\right],
$$

where: $\Omega=q B_{0} / m \gamma c$ is the particle's Larmor frequency; $\boldsymbol{k}$ is the wave-vector of the turbulent fluctuations; $k_{\|} \equiv|\boldsymbol{k}| \cos \alpha_{\text {wave }}$ is its field-aligned component ( $\alpha_{\text {wave }}$ being the angle between $\boldsymbol{k}$ and $\left.\boldsymbol{B}_{0}\right) ; \omega=\omega(\boldsymbol{k})$ the associated fluctuations' frequency. We notice that, in Equation (1), the power spectrum of the fluctuations has been explicitly split into its Alfvénic $\left(I^{\mathrm{A}}\right)$ and magnetosonic $\left(I^{\mathrm{M}}\right)$ contributions. Finally, $R_{n}$ represents a function that "resonantly" selects fluctuations whose frequency, in a reference frame that streams along $\boldsymbol{B}_{0}$ with the particle $\left(\omega^{\prime} \equiv \omega-k_{\|} v_{\|}\right)$, is either zero ( $n=0$; Landau-like wave-particle interaction) or matching a multiple of the particle gyro-frequency ( $n=1,2,3, \ldots$; gyro-resonant wave-particle interaction).

In the standard QLT treatment of purely Alfvénic turbulence, this function is a Dirac $\delta$ function. Here, instead, we include the fact that the modulus $|\boldsymbol{B}|=\left|\boldsymbol{B}_{0}+\delta \boldsymbol{B}\right|$ may not be spatially homogeneous. As a result, following [13], we adopt a broadened resonance function of the type $R_{n}\left(k_{\|} v_{\|}-\omega+n \Omega\right)=\frac{\sqrt{\pi}}{\left|k_{\|}\right| v_{\perp} M_{\mathrm{A}}^{1 / 2}} \exp \left[-\frac{\left(k_{\|} v \mu-\omega+n \Omega\right)^{2}}{k_{\|}^{2} v^{2}\left(1-\mu^{2}\right) M_{\mathrm{A}}}\right]$, where the broadening is determined by the level of the fluctuations through the Alfvénic Mach number at the injection scale $L, M_{\mathrm{A}} \sim\left(\delta B / B_{0}\right)_{L}$. We notice that $R_{n}$ reduces to a Dirac $\delta$-function, $R_{n} \rightarrow \pi \delta\left(k_{\|} v_{\|}-\omega+n \Omega\right)$, in the limit $M_{\mathrm{A}} \rightarrow 0$.

For the turbulent power spectra $I^{\mathrm{A}, \mathrm{M}}$, we follow the prescription given in $[16,17]$ and consider the two-point correlation tensors between the fluctuation components, $\left\langle\delta B_{i}(\boldsymbol{k}) \delta B_{j}^{*}\left(\boldsymbol{k}^{\prime}\right)\right\rangle / B_{0}^{2}=$ $\delta^{3}\left(\boldsymbol{k}-\boldsymbol{k}^{\prime}\right) \mathcal{M}_{i j}$, where $I^{\mathrm{A}, \mathrm{M}}=\sum_{i=1}^{3} \mathcal{M}_{i i}$ and the spectral scalings are resulting from simulations [5, 6]. In particular, we use $I^{\mathrm{A}, \mathrm{S}}\left(k_{\|}, k_{\perp}\right)=C_{a}^{\mathrm{A}, \mathrm{S}} k_{\perp}^{-10 / 3} \exp \left(-L^{1 / 3} k_{\|} / k_{\perp}^{2 / 3}\right)$ for the Alfvén and slow modes, consistently with the usual GS95 spectrum, while for fast modes we use the isotropic spectrum $I^{\mathrm{F}}(k)=C_{a}^{\mathrm{F}} k^{-3 / 2}$.

As a final comment on the calculation of $D_{\mu \mu}$, the integral has to be performed up to the truncation scale $k_{\max }$ of the turbulence, namely up to the wave-number at which the cascading timescale equals the dissipation scale, as discussed in [18]. 

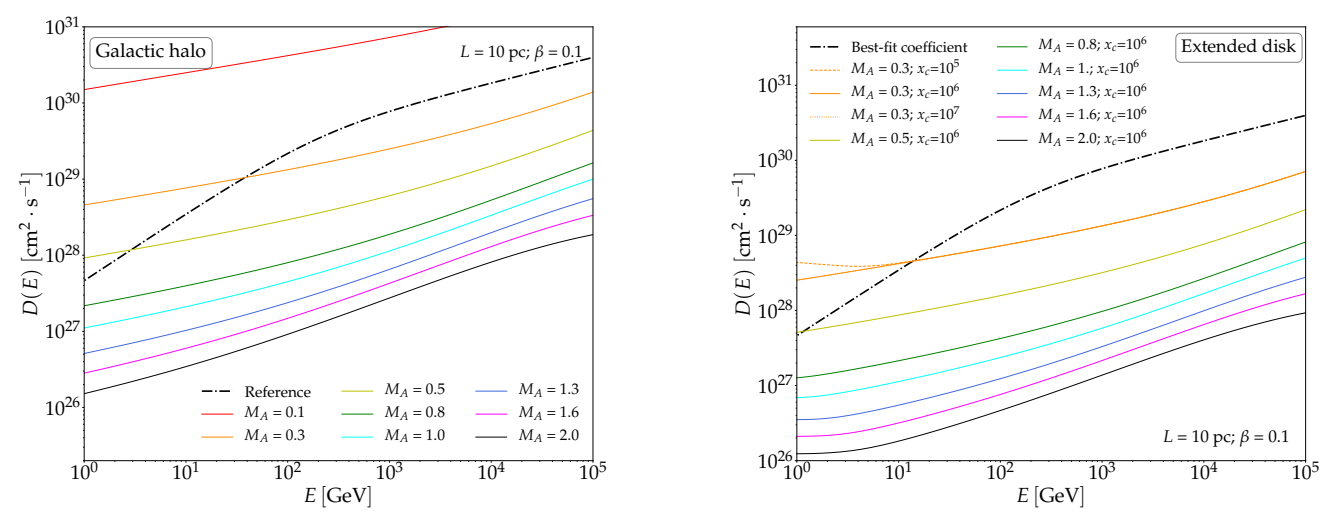

Figure 1: We show the diffusion coefficients associated to the pitch-angle scattering onto MHD fluctuations as a function of the rigidity in the halo and in the extended disk, with $L_{\mathrm{inj}}=10 \mathrm{pc}$ and plasma $\beta=0.1$, for several values of $M_{\mathrm{A}}$. Black dashed line: reference diffusion coefficient taken from [20], designed to reproduce the AMS-02 data on both primary and secondary species.

Once all the contributions from the three modes are computed, we can obtain the parallel spatial diffusion coefficient $D$ as a function of the (dimensionless) particle rigidity $R=L^{-1}|v| / \Omega$ :

$$
D(R)=\frac{1}{4} \int_{0}^{\mu^{*}} d \mu \frac{v^{2}\left(1-\mu^{2}\right)^{2}}{D_{\mu \mu}^{\mathrm{M}, \mathrm{T}}(R)+D_{\mu \mu}^{\mathrm{M}, \mathrm{G}}(R)+D_{\mu \mu}^{\mathrm{A}, \mathrm{G}}(R)},
$$

where $\mu^{*}$ is the the largest $\mu \in[0,1]$ for which a particle travels in the diffusive regime.

We select the diffusing particles according to the following criterion: if $\tau_{\text {stream }} \sim L_{\mathrm{H}, \mathrm{D}}^{\prime} / \nu$ is the streaming timescale of a CR across a distance $L_{\mathrm{H}, \mathrm{D}}^{\prime}$, the pitch-angle scattering time of such particle, $\tau_{\mu \mu} \sim\left(1-\mu^{2}\right) / D_{\mu \mu}$, (i.e., the timescale between two consecutive pitch-angle scattering events) must be much shorter than $\tau_{\text {stream }}: \frac{\tau_{\mu \mu}}{\tau_{\text {stream }}} \sim \frac{v}{L_{\mathrm{H}, \mathrm{D}}^{\prime}} \frac{\left(1-\mu^{2}\right)}{D_{\mu \mu}} \ll 1$, where we choose $L_{\mathrm{H}, \mathrm{D}}^{\prime}=L_{\mathrm{H}, \mathrm{D}} / 5$.

Based on Equation (2), in Figure 1 we plot the diffusion coefficients for several values of the Alfvénic Mach number $M_{\mathrm{A}}$, and show how they are shaped by the parameters involved in the calculation. They are plotted in the two zones mentioned in the introduction: the "extended disk", characterized by the presence of warm ionized hydrogen and a collisional — as well as collisionless - damping for the turbulent waves, and the "diffuse halo", with a low-density plasma characterized by a negligible Coulomb scattering rate, i.e. a collisionless damping exclusively. The calculations are carried out using the code in $[19]^{3}$.

First of all, we notice that (i) the high-rigidity slope predicted by the theory is perfectly compatible with the high-rigidity slope of the reference diffusion coefficient fitted on CR data, and (ii) the theory predicts a clear departure from a simple power law for all values of the relevant parameters; however, this departure does not describe the low-energy downturn of the reference coefficient, that reflects the behaviour of AMS-02 data. Hence, we may argue that the theory provides a correct description of CR confinement above $\simeq 200 \mathrm{GV}$, while an accurate low-energy treatment requires additional theoretical arguments.

The normalization spans several orders of magnitude, mainly governed by the value of $M_{\mathrm{A}}$, and reasonable values of this parameter are associated to the correct normalization. In particular

\footnotetext{
${ }^{3}$ https://github.com/ottaviofornieri/Diffusion_MHD_modes
} 


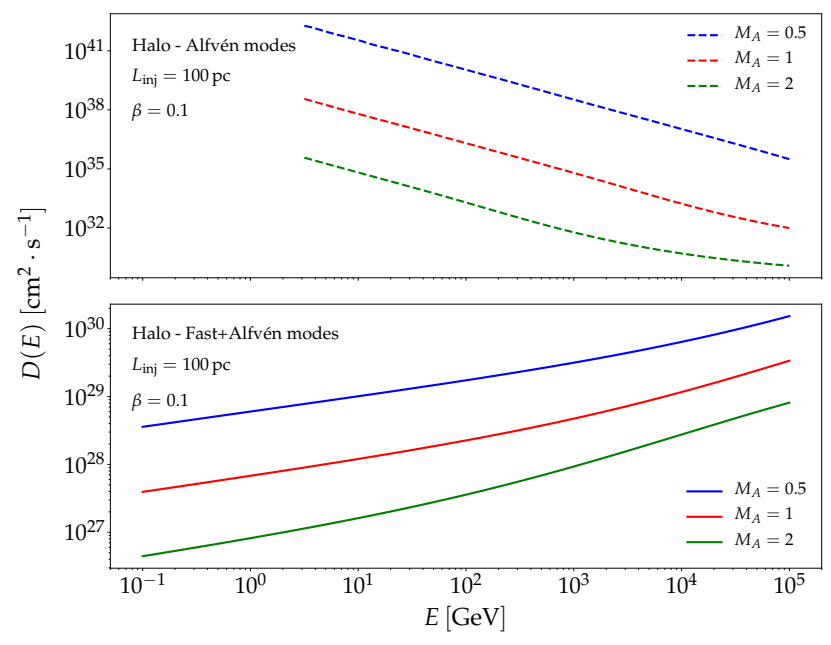

Figure 2: We show the total diffusion coefficient with fast modes included in the calculation (lower panel) compared to the case in which only Alfvén fluctuations are taken into account (upper panel). Alfvénic turbulence is not efficient in confining Galactic CRs, due to the anisotropy of the cascade.

$D(E)$ is a decreasing function of the Alfvénic Mach number: this is due to the fact that an increased level of turbulence results in a more effective CR scattering rate.

The most relevant difference between the behaviour of $D(E)$ in the halo (left panel) and in the extended disk (right panel) is the minimum in the low-energy domain $\left(\rho_{\min } \sim 50-100 \mathrm{GV}\right)$ in the latter case, which can be explained by considering that, in the extended disk, both collisionless and collisional damping mechanisms are at play, the latter dominating for particles with low Larmor radius, i.e. at low energy.

Another aspect that deserves a special treatment is connected to the role of the Alfvén modes in the resulting diffusion coefficients. In Figure 2 we show the diffusion coefficient when fast modes are included (lower panel) compared to the case where only Alfvén modes enter the calculation (upper panel). As it can be immediately seen, the normalization of $D(E)$ for the Alfvén-only case spans from just a few up to $\sim 15$ orders of magnitude more than the case where fast modes are included. Considering the average residence time of CRs in the Galaxy to be $\tau_{\text {esc }} \simeq 15 \mathrm{Myr}$, this implies $\langle D\rangle=\frac{L_{\mathrm{H}}^{2}}{2 \tau_{\mathrm{esc}}} \sim 10^{30} \mathrm{~cm}^{2} / \mathrm{s}$ to be the highest possible diffusion coefficient allowing CRs to be confined in the Galaxy. Therefore, if only Alfvén modes were present, local CR abundances could not be reproduced. Also, the Alfvén-only case shows a declining trend with particle rigidity.

Both features derive from the anisotropic behavior of the alfvénic cascade. Indeed Alfvén modes evolve on the isosurfaces identified by $k_{\|} \propto k_{\perp}^{2 / 3}$ [7]. This relation implies that turbulent eddies are spatially elongated along $\boldsymbol{B}$, or, equivalently, that in the momentum space they are elongated in the perpendicular direction. Thus, the majority of the power goes into a $k_{\perp}$ cascade. This leaves very little power (i.e. scattering efficiency) to the cascade in parallel wave numbers $k_{\|}$ that, according to the adopted resonance function, is the component involved in the wave-particle interaction. Since $k_{\|} \sim \ell_{\|}^{-1}$, particles with small rigidity and small Larmor radius get weakly confined, while high-energy CRs scatter more efficiently. As a result, an efficient wave-particle scattering with Alfvén modes can occur only at high energies, that resonate with scales not too far 

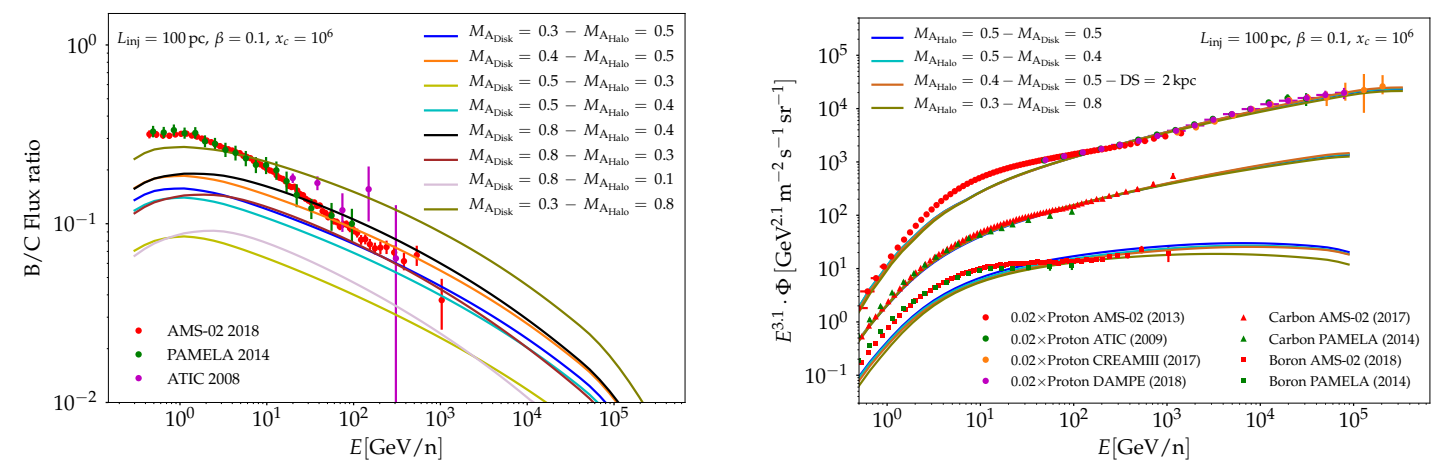

Figure 3: Left panel: theoretical prediction for the $\mathrm{B} / \mathrm{C}$ within a general setup where extended disk and halo exhibit different values of $M_{\mathrm{A}}$. Right panel: theoretical prediction for the $H, C$ and $B$ fluxes for a few selected combinations of the parameters of interest. The primary injection spectrum is tuned to fit the data above $200 \mathrm{GeV}$.

from the injection scale, where the anisotropic nature of the cascade has not become significant yet. This is visible in the change of slope in $D(E)$ for the larger Mach numbers of Figure 1, in the high-energy domain $\left(E \geq 10^{4} \mathrm{GeV}\right)$, where Alfvén modes play a role that is comparable to that of the fast isotropic modes.

In conclusion, for the injection scale $L_{\text {inj }}$ and Alfvénic Mach number we are considering throughout this work, anisotropy of the Alfvén cascade always plays a key role and therefore cannot efficiently confine cosmic rays, while the fast magnetosonic cascade is able to induce a very efficient pitch-angle scattering rate.

\section{Phenomenological implications of the theory}

In this Section we compare the propagated CR spectrum, obtained adopting the diffusion coefficients discussed above, with the most relevant CR observables. We implement the coefficients - obtained by means of Equation (2) - in the DRAGON2 code and solve the usual diffusion-loss equation that characterizes the propagation of high-energy charged particles in the Galaxy [21]. We refer to the DRAGON2 technical paper [22] for the implementation of such equation in the code.

A key observable in the context of CR phenomenology is the Boron-over-Carbon $(\mathrm{B} / \mathrm{C})$ flux ratio. In fact, Boron is entirely secondary and is mostly produced in spallation reactions involving heavier species (including Carbon): therefore, the ratio between those two fluxes constrains the residence time of the primaries and, in turn, the confining power of the theory.

Given these considerations, in the left panel of Figure 3 we focus on this observable, and pay attention to the dependence of the computed B/C flux ratio on the Alfvénic Mach number of pre-existing MHD turbulence, $M_{\mathrm{A}}$, which was shown to play a key role in the overall normalization of the transport coefficients. We scan over this parameter, and find that larger values of $M_{\mathrm{A}}$ are associated with a significant over-production of Boron, especially at high energies, due to the high efficiency of the confinement characterizing large- $M_{\mathrm{A}}$ scenarios. 
We remark again that in all cases the high-energy slope is correctly reproduced, while the low-energy domain suggests an extra grammage possibly associated to a different confinement mechanism (not captured by the theory presented here) that starts to dominate below $\sim 200 \mathrm{GeV}$.

We now widen our perspective and consider a variety of secondary and primary species, as measured by the AMS-02 Collaboration [23-25]. The most relevant feature revealed by such spectra is a progressive hardening above $\sim 200 \mathrm{GV}$. Such hardening is twice as large in the secondary species with respect to the primaries, suggesting a transport origin for the feature [26]. For what concerns its physical origin, it may be attributed to the transition between a diffusive regime dominated by scattering against self-generated waves, and a regime regulated by pre-existing turbulent fluctuations $[27,28]$.

Motivated by these considerations, and given the aspects highlighted in the study of $\mathrm{B} / \mathrm{C}$, we aim at providing a comprehensive picture of the high-energy portion of the spectrum, above the aforementioned break, where the confinement due to scattering onto isotropic fast-magnetosonic turbulence should be the dominant physical mechanism. In the right panel of Figure 3, we show a particular realization that satisfies all the experimental constraints in the high-energy regime. We show that we can consistently reproduce all the observed data above the $200 \mathrm{GV}$ spectral feature, by assuming a reasonable injection slope $(\gamma=2.3)$ and propagating the particles within the setup described above. Again, the "excess" at low energy cannot be reproduced within the framework discussed in the present work.

\section{Summary}

In this work we have presented for the first time a comprehensive phenomenological study adopting a (weakly) non-linear extension of the QLT of CR scattering onto MHD fluctuations.

We have considered a state-of-the-art description of pitch-angle scattering associated to the three MHD cascades, i.e., decomposed into a (anisotropic) cascade of Alfvénic fluctuations, and slow and fast (isotropic) magnetosonic modes. We have studied the physical problem of the interaction of a charged, relativistic particle with such modes and we have computed the associated transport coefficients from first principles - by identifying a set of parameters that characterize the ISM and have significant impact on our result (i.e. the $M_{\mathrm{A}}$, the plasma $\beta$, and some parameters that describe the damping processes in different environments) - and presented a complete phenomenological study of the dependence of the diffusion coefficients on those parameters.

Then, we implemented the coefficients in the DRAGON2 code and tested the theory against current experimental data, with particular focus on the extremely accurate AMS-02 dataset. We found that the high-energy behaviour of the $D(E)$ nicely matches the secondary-over-primary slope in that regime, and a reasonable range of the aforementioned parameters allowed us to reproduce the correct normalization as well, without invoking any ad hoc tuning. Overall, we found a natural agreement with all CR channels within a reasonable choice of both the ISM parameters governing the transport process, and other parameters (e.g. injection slope) that characterize our setup.

The theory is therefore adequate to describe the microphysics of Galactic CR confinement in the high-energy domain, in particular above the $200 \mathrm{GeV}$. Below such energy, the steeper spectrum seems to require additional physical effects, for instance a self-confinement due to the enhancement of streaming instabilities. 


\section{References}

[1] S. Gabici et al., Int. J. Mod. Phys. D Vol. 28 No. 15 (2019).

[2] J. R. Jokipii, Astrophys. J. 146 (1966).

[3] K. Hasselmann and G. Wibberenz, Astrophys. J. 162 (1970).

[4] N. Tomassetti, Astrophys. J. Lett. Vol. 752 L13 (2012).

[5] J. Cho et al., Astrophys. J. Vol. 564 291-301 (2002).

[6] J. Cho and A. Lazarian, Phys. Rev. Lett. 88245001 (2002).

[7] P. Goldreich and S. Sridhar, Astrophys. J. Vol. 438 (1995).

[8] V. S. Ptuskin et al., Astrophys. J. Vol. 642 902-916 (2006).

[9] A. Barnes, Physics of Fluids Vol. 9 1483-1495 (1966).

[10] B. Chandran, Phys. Rev. Lett. Vol. 85 4656-4659 (2000).

[11] H. J. Völk, Ap\&SS Vol. 25 No. 2 (1973).

[12] H. J. Völk, Reviews of Geophysics and Space Physics Vol. 13 547-566 (1975).

[13] H. Yan and A. Lazarian, Astrophys. J. Vol. 673942 (2008).

[14] O. Fornieri et al., MNRAS 502, 5821-5838 (2021).

[15] R. Kulsrud and W. P. Pearce, Astrophys. J. Vol. 156445 (1969).

[16] R. Schlickeiser, Cosmic Ray Astrophysics, Springer-Verlag Heidelberg (2002).

[17] H. Yan and A. Lazarian, Phys. Rev. Lett. Vol. 89281102 (2002).

[18] H. Yan and A. Lazarian, Astrophys. J. Vol. 614 757-769 (2004).

[19] O. Fornieri, https://doi.org/10.5281/zenodo.4250807 (2020).

[20] O. Fornieri et al., JCAP 02009 (2020).

[21] V. L. Ginzburg and S. I. Syrovatskii, The Origin of Cosmic Rays, New York (1964).

[22] C. Evoli et al., JCAP Vol. 2015 (2017).

[23] M. Aguilar et al., Phys. Rev. Lett. Vol. 114171103 (2015).

[24] M. Aguilar et al., Phys. Rev. Lett. Vol. 117231102 (2016).

[25] M. Aguilar et al., Phys. Rev. Lett. Vol. 121051103 (2018).

[26] Y. Génolini et al., Phys. Rev. Lett. Vol. 119241101 (2017).

[27] A. J. Farmer and P. Goldreich, Astrophys. J. Vol. 604 671-674 (2004).

[28] P. Blasi et al., Phys. Rev. Lett. Vol. 109061101 (2012). 